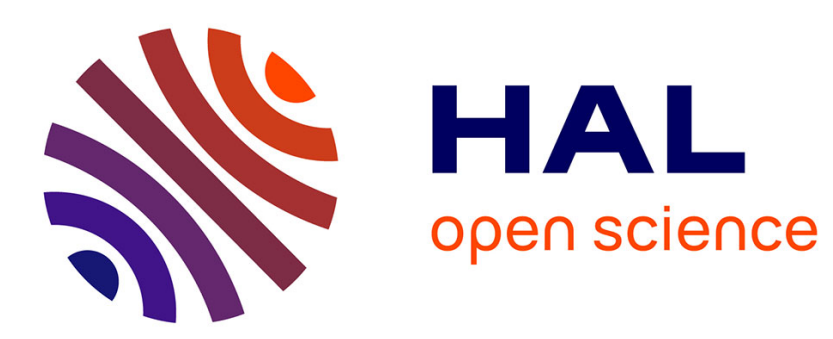

\title{
Modeling of photon trapping effects in high-density divertor plasmas
}

J. Rosato, M. Koubiti, Y. Marandet, R. Stamm

\section{To cite this version:}

J. Rosato, M. Koubiti, Y. Marandet, R. Stamm. Modeling of photon trapping effects in high-density divertor plasmas. Journal of Quantitative Spectroscopy and Radiative Transfer, 2020, 247, pp.106949. 10.1016/j.jqsrt.2020.106949 . hal-03215650

\section{HAL Id: hal-03215650 \\ https://hal.science/hal-03215650}

Submitted on 11 May 2021

HAL is a multi-disciplinary open access archive for the deposit and dissemination of scientific research documents, whether they are published or not. The documents may come from teaching and research institutions in France or abroad, or from public or private research centers.
L'archive ouverte pluridisciplinaire HAL, est destinée au dépôt et à la diffusion de documents scientifiques de niveau recherche, publiés ou non, émanant des établissements d'enseignement et de recherche français ou étrangers, des laboratoires publics ou privés. 


\title{
Modeling of photon trapping effects in high-density divertor plasmas
}

\author{
J. Rosato, M. Koubiti, Y. Marandet, and R. Stamm \\ Aix-Marseille Université, CNRS, PIIM UMR 7345, F-13397 Marseille, France
}

\begin{abstract}
In divertor plasmas at high density regimes, typically with $N_{e}$ larger than $10^{14} \mathrm{~cm}^{-3}$, the photons emitted in the resonance line of the hydrogen isotopes have a mean free path that can be as short as one millimeter. They provide an additional source of excited atoms, which can be ionized more easily; a consequence of this is that the whole ionization-recombination balance of the divertor plasma can be altered. In this work, we investigate the influence of the spectral line profile on the photoexcitation rate. A kinetic Monte Carlo solver is applied to the transport of photons in slab geometry. The relative contribution of the Doppler and Zeeman effects on the line profile is examined. In the framework of ITER nuclear phase preparation, we also examine the influence of deuterium and tritium line shape overlapping and we address the transport of the helium resonance line radiation.
\end{abstract}

Keywords:

\section{Introduction}

In the last two decades, investigations of line radiation trapping effects carried out using dedicated transport codes $[1,2,3,4,5]$ have indicated the possibility of an alteration of the ionization-recombination balance in tokamak divertor plasmas. At high atomic density conditions, typically larger than $10^{14} \mathrm{~cm}^{-3}$, the photon mean free path can be smaller than $1 \mathrm{~mm}$ near the first Lyman transitions $(n=1 \rightarrow 2, n=1 \rightarrow 3$ etc.), indicating that the plasma is opaque to these lines. The preparation of operation and interpretation of large-scale fusion reactor devices (ITER, DEMO) requires accurate

Email address: joel.rosato@univ-amu.fr () 
transport models for the plasma edge able to account for neutral and charged particles, together with line radiation, in a self-consistent fashion. In this work, following previous investigations [6], we investigate the influence of the spectral line profile on the deuterium Lyman $\alpha(n=1 \rightarrow 2)$ photoexcitation rate. A kinetic Monte Carlo solver is applied to the transport of photons in high density divertor conditions. For the sake of simplicity in discussions, an ideal slab geometry is considered. We examine the relative contribution of the Doppler and Zeeman effects on the line profile, and the consequence of these mechanisms on the photoexcitation rate. In the framework of the ITER nuclear phase preparation, we also address the photon trapping in the resonance line of tritium, and, in particular, the role of overlapping with the deuterium Lyman $\alpha$ spectrum in the photoexcitation process. The reabsorption of the helium resonance line $\left(1 \mathrm{~s}^{2} \rightarrow 1 \mathrm{~s} 2 \mathrm{p}\right)$ photons, which is expected to be important, is also examined.

\section{Kinetic modeling of line radiation transport}

We give hereafter a summary of relevant formulas employed in the modeling of radiation transport; more details can be found in previous articles $[6,7]$. For our purposes it will be sufficient to focus on the time-independent radiative transfer problem. Consider a gas of photons interacting with a set of atoms through emission and absorption processes; the radiative transfer equation in stationary regime reads

$$
\vec{n} \cdot \vec{\nabla} I=\eta-\kappa I,
$$

where $I$ is the specific intensity and $\eta$ and $\kappa$ are the emission and extinction coefficients:

$$
\begin{aligned}
\eta & =\sum_{u l} \frac{h \nu_{u l}}{4 \pi} N_{u} A_{u l} \psi_{u l}, \\
\kappa & =\sum_{u l} \frac{h \nu_{u l}}{4 \pi}\left(N_{l} B_{l u} \phi_{u l}-N_{u} B_{u l} \psi_{u l}\right) .
\end{aligned}
$$

The $u$ and $l$ subscripts refer to the upper and lower levels of the transition, respectively; $\nu_{u l}$ is the corresponding Bohr frequency; $A_{u l}, B_{l u}$, and $B_{u l}$ are the Einstein coefficients; $N_{u}, N_{l}$ are the densities of atoms in the upper and lower states; and $\psi_{u l}, \phi_{u l}$ are the emission and absorption line shapes normalized such that $\int d \nu \int d \Omega \phi_{u l}=4 \pi=\int d \nu \int d \Omega \psi_{u l}$. They denote the 
conditional probability density function of emitting or absorbing a photon at the frequency $\nu$, given that the event of emission or absorption is already known to occur at a certain position. These functions depend on the radiation propagation direction $\vec{n}$ due to the magnetic field (Zeeman effect). The fact that the stimulated emission is treated as a negative part in the absorption is only a matter of convention, which allows one to write the radiative transfer equation in the compact form given in Eq. (1). The emission coefficient $\eta$ describes the spontaneous emission from atoms excited due to both collisions and photon absorption; it includes a scattering term that renders the transfer equation integral. In the case where the photon states prior to and after a scattering process are independent, one deals with the so-called complete redistribution assumption and the emission and absorption line shapes are identical. We will work within this approximation in the following; partial redistribution effects can be addressed through the use of two-photon line shape functions, e.g. [8]. For simplicity sake in discussions, we will also neglect feedback of the radiation field on the atomic populations; a more elaborate model requires the solving of a collisional-radiative model, e.g. [3], which renders the problem non-linear.

Once the radiative transfer equation is solved, one can evaluate the photoexcitation rate corresponding to transition $l \rightarrow u$ using the following relation:

$$
W_{l u}=B_{l u} \int d \nu \int \frac{d \Omega}{4 \pi} \phi_{u l} I \equiv B_{l u} \bar{I}_{u l} .
$$

This rate can be inferred from line ratio measurement (this method was precisely used on Alcator C-Mod for demonstrating the presence of Lyman line opacity[9]). In the framework of plasma edge transport modeling, it serves as an input in a collisional-radiative model. At low density regimes where the medium is optically thin, the photon trapping effect is negligible and the excitation is mainly due to collisions with electrons; in this framework, there is no need for calculating the photoexcitation rate (4) nor for solving the radiative transfer equation (1). At high density regimes, the relevance of photon trapping can be evaluated by using the ratio between the mean free path of photons having an energy $h \nu$ equal to the Bohr transition energy $h \nu_{u l}$ and the characteristic plasma size. By construction, this ratio is very small in optically thick conditions. The photon mean free path can be evaluated by using the inverse of the extinction coefficient $\kappa_{\nu}$. Figure 1 shows a set of representative values for the resonance line of deuterium and tritium (Lyman $\alpha, n=1 \rightarrow 2$ ), and the resonance line of helium $\left(1 \mathrm{~s}^{2} \rightarrow 1 \mathrm{~s} 2 \mathrm{p}\right)$. 


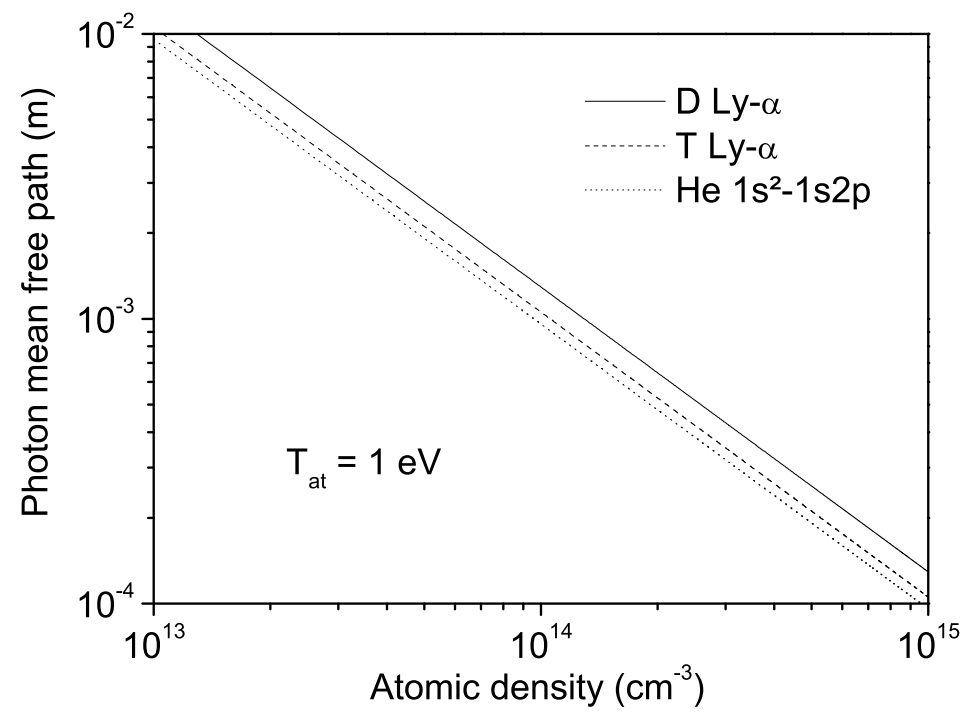

Figure 1: Plot of the photon mean free path relative to the first transition line of deuterium $(\lambda=121.53 \mathrm{~nm}$, e.g. www.nist.gov $)$, tritium $(\lambda=121.52 \mathrm{~nm})$, and helium $(\lambda=58.43$ $\mathrm{nm}$ ), in terms of the atomic density. Here, a focus is put on resonant photons, i.e., those having an energy strictly equal to the Bohr energy $h \nu_{u l}$. The atomic temperature entering the mean free path formula has been set equal to $1 \mathrm{eV}$. As can be seen, the mean free path is smaller than $1 \mathrm{~mm}$ at high density conditions, indicating the relevance of the photon trapping effect.

The line shape entering the $\kappa_{\nu}$ coefficient has been evaluated here assuming a Gaussian Doppler broadening function, i.e., $\phi_{u l}\left(\nu_{u l}\right) \equiv 1 / \Delta \nu_{D} \sqrt{\pi}$ where $\Delta \nu_{D}=\nu_{u l} v_{0} / c$ and where the quantity $v_{0}=\sqrt{2 k_{B} T_{a t} / m_{a t}}$ stands for the atomic thermal velocity. A value of $1 \mathrm{eV}$, which is representative of cold and dense divertor plasma conditions, has been assumed for the temperature. As can be seen in the figure, the photon mean free path decreases to values smaller than $1 \mathrm{~mm}$ if the atomic density is larger than $10^{14} \mathrm{~cm}^{-3}$, indicating that the plasma is optically thick to the line radiation. Note that a similar trend is expected if one plots the mean free path in terms of $1 / \sqrt{T_{a t}}$ due to the temperature dependence of the Doppler broadening function. 


\section{Influence of line broadening mechanisms on the photoexcitation rate}

The photoexcitation rate (4) depends on the spectral line shape function $\phi_{u l}$, both directly and implicitly through the presence of the specific intensity $I$ in the integral. For the resonance lines of neutrals in tokamak edge plasma conditions, the line shape function is usually dominated by the Doppler broadening, but the Zeeman effect can provide a significant additional broadening, in particular at strong magnetic fields (see Fig. 2). At extremely high density regimes $\left(N_{e} \sim 10^{15} \mathrm{~cm}^{-3}\right)$, there is also a significant contribution of Stark broadening; this concerns the hydrogen isotope lines in particular because the energy levels have a degeneracy in the orbital quantum number $l$, which renders them more sensitive to the plasma's microscopic electric field (this argument stems from basic quantum-mechanical perturbation theory). In general, the modeling of Stark broadening requires an elaborated treatment, involving dedicated numerical codes. We will not consider this effect in the following; for specific works in the framework of dense divertor plasmas, e.g., see $[10,11,6]$.

We have applied a kinetic Monte Carlo solver to the radiative transfer equation (1) for the deuterium and helium resonance lines, and we have evaluated the corresponding photoexcitation rate. Details on the calculation method can be found in [7]. For simplicity sake, a homogeneous plasma slab has been considered, and the density of emitters has been artificially set equal to one (practically, this means that the absolute value of the photoexcitation rate is expressed in arbitrary units; see appendix for an estimate in absolute units). The density of atoms in ground state has been set equal to $10^{14}$ $\mathrm{cm}^{-3}$ and their temperature has been set equal to $1 \mathrm{eV}$. The slab size has been set to $2 \mathrm{~mm}$, i.e., a value comparable to the photon mean free path. The magnetic field has been assumed perpendicular to the slab surface. The result is shown in Fig. 3. As can be seen in the figure, there is a significant decrease of the photoexcitation rate if the Zeeman effect is taken into account. Qualitatively, this decrease is due to the additional line broadening provided by the Zeeman effect; the latter yields a smaller line shape maximum (from area-normalization) and this results in a decrease of the extinction coefficient maximum.

A similar result is expected if there is an overlapping between neighboring lines corresponding to different hydrogen isotopes. Figure 4 illustrates this

point. The Lyman $\alpha$ line of deuterium and tritium has been considered as an 

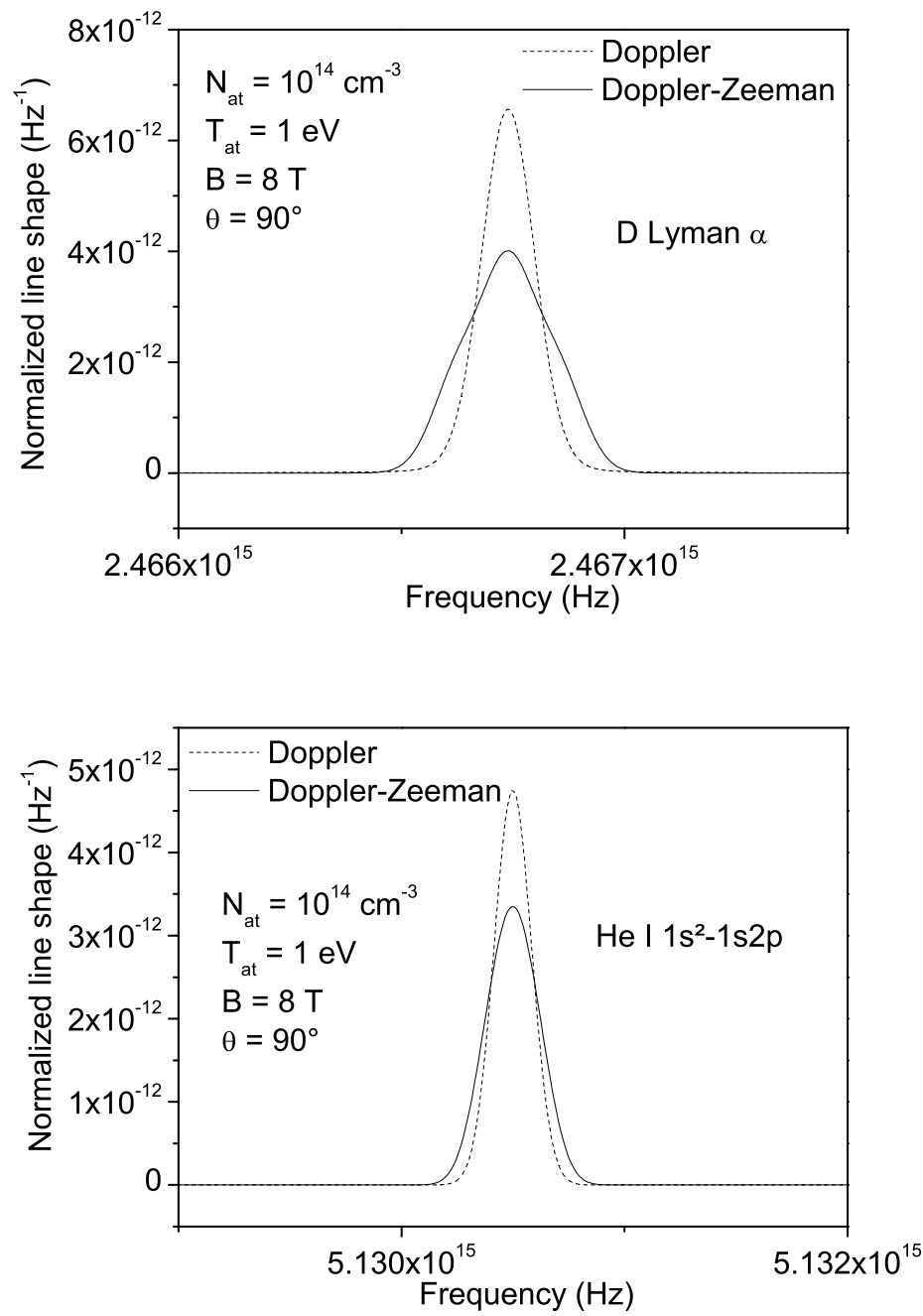

Figure 2: Plot of the resonance line shapes of deuterium and helium, calculated retaining Doppler broadening only (dashed line) and retaining both Doppler broadening and Zeeman effect (solid line). A photon direction perpendicular to the magnetic field has been assumed. The value of 8 teslas assumed for the magnetic field is representative of that inside the inner divertor of ITER [12]. The Zeeman effect, which splits the line into three components, results in an overall additional broadening of the line.

example, which is representative of ITER plasmas during the nuclear phase. 

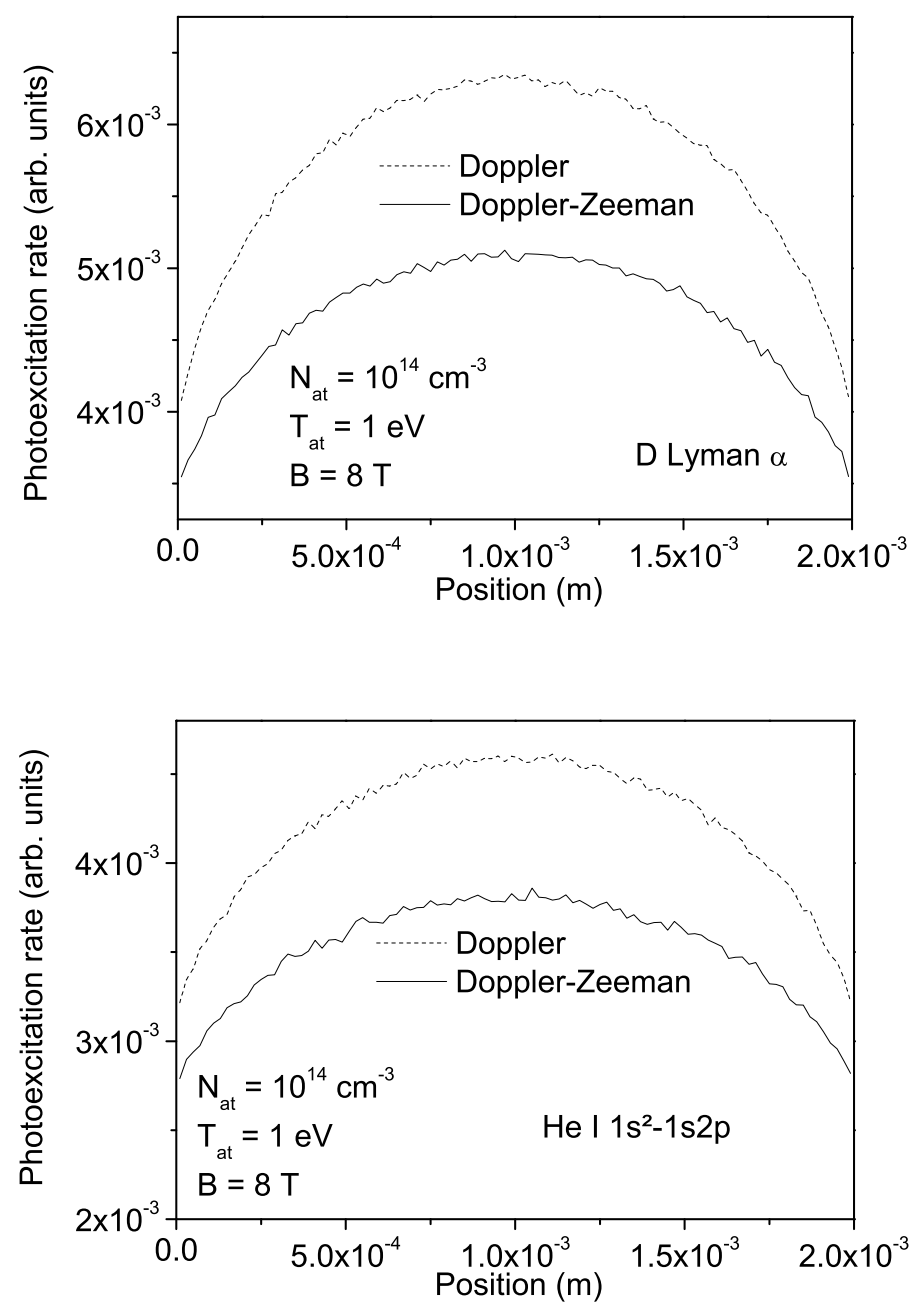

Figure 3: The photoexcitation rate is sensitive to the line broadening mechanisms. Here, a Monte Carlo calculation has been performed for the resonance lines of deuterium and helium, assuming a homogeneous slab of $2 \mathrm{~mm}$. The Zeeman effect, which broadens the spectral line, results in a decrease of the photoexcitation rate.

The deuterium and tritium Lyman $\alpha$ frequencies are close enough, so as to make the spectral profiles overlapping. This results in a common apparent line shape broader, with a smaller maximum, than that in a pure deuterium 

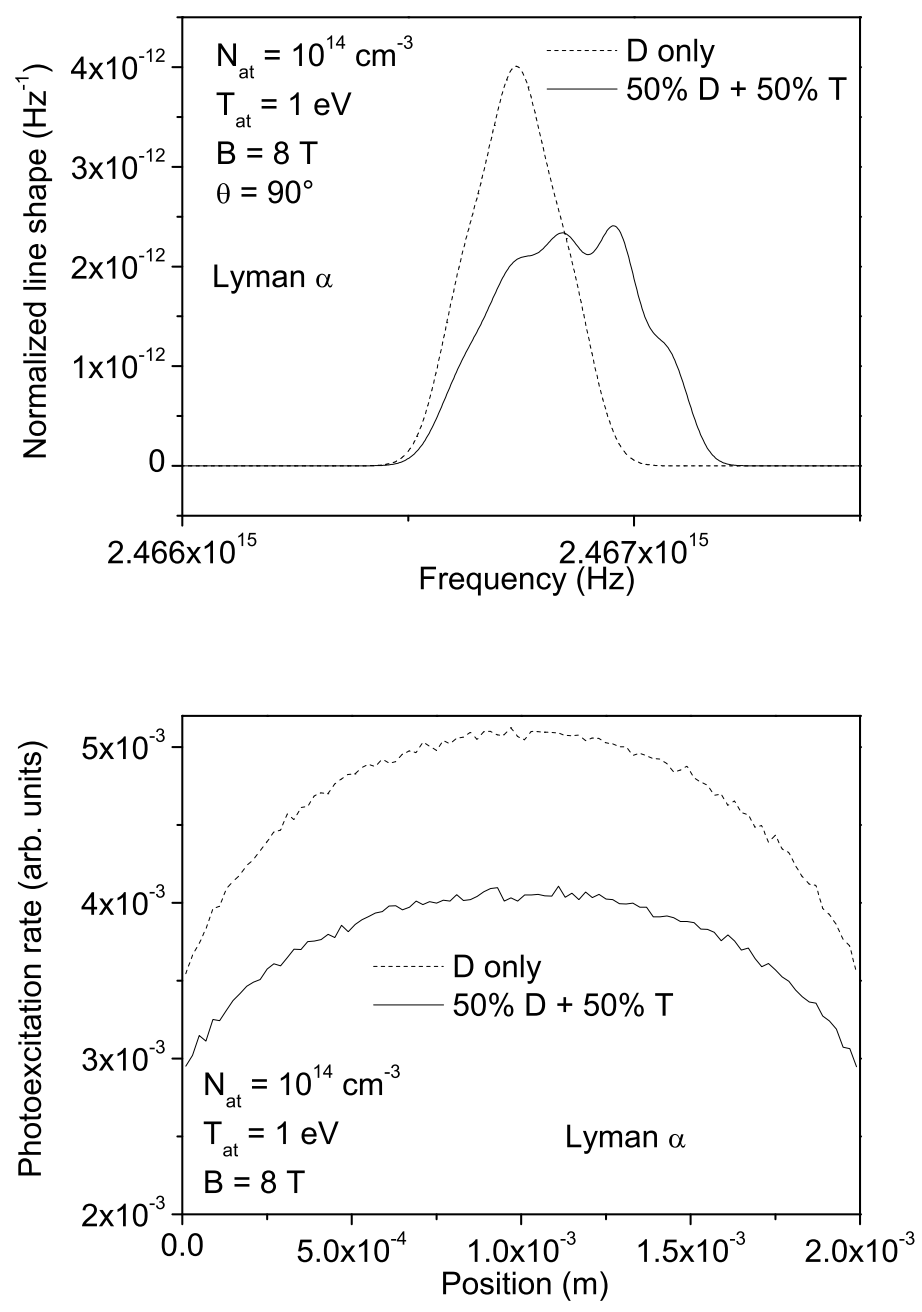

Figure 4: The photoexcitation rate is also sensitive to the overlapping between two neighboring lines. Here, a calculation has been performed in the case of plasma with an equal amount of deuterium and tritium.

plasma. A way to account for this effect consists in using a common effective line shape function in terms of the tritium abundance $\alpha=N_{T} /\left(N_{D}+N_{T}\right)$ as $\phi_{u l}^{\text {eff }} \equiv(1-\alpha) \phi_{u l}^{D}+\alpha \phi_{u l}^{T}$, where $\phi_{u l}^{D}$ and $\phi_{u l}^{T}$ are the deuterium and tritium line shape function, respectively [formally: the effective line shape is defined 
in such a way that the quantity $N_{D} \phi_{u l}^{D}+N_{T} \phi_{u l}^{T}$ that enters the emission and extinction coefficients is identical to $\left.\left(N_{D}+N_{T}\right) \phi_{u l}^{\text {eff }}\right]$. The plot on the left side of Fig. 4 shows the effective line shape in the presence of $50 \%$ of tritium, compared to that obtained assuming pure deuterium plasma, and the plot on the right side shows the rate corresponding to deuterium photoexcitation. The Monte Carlo solver has been applied using the same conditions as above. As can be seen in the figure, the photoexcitation rate is significantly reduced when the tritium Lyman $\alpha$ line radiation is retained.

\section{Conclusion}

We have applied a kinetic model to the transport of line radiation in optically thick dense divertor plasmas. Both the hydrogen isotopes and helium have been considered. Numerical calculations in ideal geometry have indicated that the photoexcitation rate corresponding to resonance transitions is sensitive to the detailed spectral profile, and in particular to the Zeeman effect. The calculation results have shown that the photoexcitation rate is less important than that expected from a model neglecting the Zeeman effect. This can be explained qualitatively in terms of the structure of the extinction coefficient and its dependence on the photon frequency. A similar effect is also expected from spectrum overlapping between neighboring hydrogen isotope lines, such as the deuterium and tritium Lyman $\alpha$ transitions. The present results are still qualitative and require complementary investigations. The feedback of the radiation field on the atomic populations, not retained here, should be accounted for in a consistent way, by using a dedicated collisional-radiative model. Other specific assumptions used in this work, such as the complete redistribution of radiation, have not yet been examined in detail and require further investigations.

\section{Acknowledgements}

This work has been carried out within the framework of the French Research Federation for Magnetic Fusion Studies.

\section{Appendix}

An estimate of the photoexcitation rate $W_{l u}$ is provided by Eq. (4), using that the radiation field is nearly Planckian near the resonance line in 


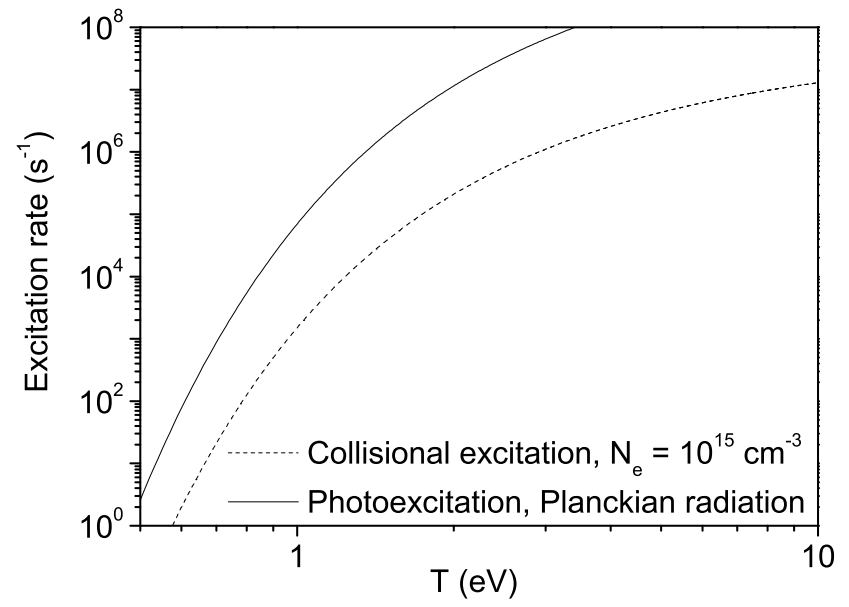

Figure 5: An estimate of the Lyman $\alpha$ photoexcitation rate assuming Planckian radiation indicates that it is larger than the collisional excitation rate, indicating the importance of radiation trapping effects in optically thick divertor plasmas.

optically thick plasma conditions. This trend was observed in previous simulations performed at high atomic density, e.g. [1]. The photoexcitation rate within this approximation reads $W_{l u} \simeq B_{l u}\left(2 h \nu_{u l}^{3} / c^{2}\right)\left[\exp \left(h \nu_{u l} / k_{B} T\right)-1\right]^{-1}$ and it involves the temperature only. Figure 5 shows a plot of this quantity for Lyman $\alpha$, compared to the collisional excitation rate. The latter has been estimated using the analytical formula reported in [13] and assuming an electron density of $10^{15} \mathrm{~cm}^{-3}$. The electron and Planck radiation temperatures have been set equal to each other. As can be seen in the figure, the photoexcitation rate is larger than the collisional excitation rate. The actual value of the photoexcitation rate is smaller (since the radiation is not Planckian), but this trend indicates that opacity effects should be accounted for in simulations of dense divertor plasmas.

\section{References}

[1] D. Reiter, S. Wiesen, M. Born, Towards radiation transport modelling in divertors with the EIRENE code, Plasma Phys. Control. Fusion 44 (2002) 1723. 
[2] H. A. Scott, M. L. Adams, Incorporating Line Radiation Effects into Edge Plasma Codes, Contrib. Plasma Phys. 44 (2004) 51.

[3] V. Kotov, D. Reiter, A. S. Kukushkin, H. D. Pacher, P. Börner, S. Wiesen, Radiation Absorption Effects in B2-EIRENE Divertor Modelling, Contrib. Plasma Phys. 46 (2006) 635.

[4] K. Hoshino, K. Sawada, R. Idei, S. Tokunaga, N. Asakura, K. Shimizu, N. Ohno, Photon Trapping Effects in DEMO Divertor Plasma, Contrib. Plasma Phys. 56 (2016) 657.

[5] E. Marenkov, S. Krasheninnikov, A. Pshenov, Multi-level model of radiation transport in inhomogeneous plasma, Contrib. Plasma Phys. 58 (2018) 578 .

[6] J. Rosato, D. Reiter, V. Kotov, Y. Marandet, H. Capes, L. GodbertMouret, M. Koubiti, R. Stamm, Progress on Radiative Transfer Modelling in Optically Thick Divertor Plasmas, Contrib. Plasma Phys. 50 (2010) 398.

[7] J. Rosato, Y. Marandet, D. Reiter, R. Stamm, Development of a hybrid kinetic-fluid model for line radiation transport in magnetic fusion plasmas, High Energy Density Phys. 22 (2017) 73.

[8] J. Rosato, D. Reiter, A. Demura, Y. Marandet, C. Mossé, R. Stamm, An analytical model for the Ly $\alpha$ redistribution function in conditions of tokamak edge plasmas, J. Phys. B: At. Mol. Opt. Phys. 41 (2008) 165701.

[9] J. L. Terry, B. Lipschultz, A. Y. Pigarov, S. I. Krasheninnikov, B. LaBombard, D. Lumma, H. Ohkawa, D. Pappas, M. Umansky, Volume recombination and opacity in Alcator C-Mod divertor plasmas, Phys. Plasmas 5 (1998) 1759.

[10] J. Rosato, Y. Marandet, H. Capes, S. Ferri, C. Mossé, L. GodbertMouret, M. Koubiti, R. Stamm, Stark broadening of hydrogen lines in low-density magnetized plasmas, Phys. Rev. E 79 (2009) 046408.

[11] J. Rosato, D. Reiter, V. Kotov, P. Börner, H. Capes, Y. Marandet, R. Stamm, S. Ferri, L. Godbert-Mouret, M. Koubiti, C. Mossé, Line 
shape modeling for radiation transport investigations in magnetic fusion plasmas, High Energy Density Phys. 5 (2009) 93.

[12] J. Rosato, V. Kotov, D. Reiter, Modelling of passive spectroscopy in the ITER divertor: the first hydrogen Balmer lines, J. Phys. B: At. Mol. Opt. Phys. 43 (2010) 144024.

[13] L. C. Johnson, Approximations for collisional and radiative transition rates in atomic hydrogen, Astrophys. J. 174 (1972) 227. 